\title{
Fluctuations and Shape of Cooperative Rearranging Regions in Glass-Forming Liquids
}

\author{
Giulio Biroli* \\ Institut de Physique Théorique (IPhT) CEA Saclay-CNRS URA 2306, \\ 91191 Gif Sur Yvette and Laboratoire de Physique Statistique, École Normale Supérieure, \\ PSL Research University, 24 rue Lhomond, 75005 Paris, France \\ Chiara Cammarota ${ }^{\dagger}$ \\ Department of Mathematics, King's College London, Strand London, WC2R 2LS United Kingdom \\ and Dipartimento di Fisica, Ed. Marconi, "Sapienza" Università di Roma, \\ P.le A. Moro 2, 00185 Roma, Italy
}

(Received 24 November 2015; revised manuscript received 15 September 2016; published 30 January 2017)

\begin{abstract}
We develop a theory of amorphous interfaces in glass-forming liquids. We show that the statistical properties of these surfaces, which separate regions characterized by different amorphous arrangements of particles, coincide with the ones of domain walls in the random field Ising model. A major consequence of our results is that supercooled liquids are characterized by two different static lengths: the point-to-set $\xi_{\mathrm{PS}}$, which is a measure of the spatial extent of cooperative rearranging regions, and the wandering length $\xi_{\perp}$, which is related to the fluctuations of their shape. We find that $\xi_{\perp}$ grows when approaching the glass transition but slower than $\xi_{\mathrm{PS}}$. The wandering length increases as $s_{c}^{-1 / 2}$, where $s_{c}$ is the configurational entropy. Our results strengthen the relationship with the random field Ising model found in recent works. They are in agreement with previous numerical studies of amorphous interfaces and provide a theoretical framework for explaining numerical and experimental findings on pinned particle systems and static lengths in glass-forming liquids.
\end{abstract}

DOI: 10.1103/PhysRevX.7.011011

\section{INTRODUCTION}

Developing a theory of the glass transition remains one of the most fundamental challenges of statistical physics and condensed matter. The interest in this problem actually goes well beyond the physics of molecular supercooled liquids. The reason is that glassy behavior is ubiquitous; it appears in a large variety of contexts, from physical systems like colloids and granular material to central problems in other branches of sciences like computer science, economics, and biology. Recent years have witnessed important and substantial progress in its understanding. Several theoretical approaches have grown in importance and in the level of detailed predictions and explanations [1]. In particular, the random first-order transition (RFOT) theory originally introduced by Kirkpatrick, Thirumalai, and Wolynes [2] has been boosted from new theoretical ideas and techniques [3-5] and innovative simulation studies [6-12]. The initial idea of Kirkpatrick, Thirumalai, and Wolynes that

\footnotetext{
giulio.biroli@cea.fr

chiara.cammarota@kcl.ac.uk
}

Published by the American Physical Society under the terms of the Creative Commons Attribution 4.0 International license. Further distribution of this work must maintain attribution to the author(s) and the published article's title, journal citation, and DOI.
Subject Areas: Materials Science, Soft Matter,

Statistical Physics supercooled liquids are in a mosaic state, a kind of microphase separated state in which the number of possible phases is huge, has been made concrete and testable in analytical computations and numerical simulations through precise and measurable definitions of the spatial extent of amorphous order, i.e., the length scale over which particles (or molecules) in supercooled liquids are arranged in an ordered fashion, even though apparently chaotic. Numerical simulations have shown that this length $\xi_{\mathrm{PS}}$, called point to set, grows upon supercooling and plays an important role in the static and dynamical behaviors. In this work, we unveil the existence of a second static length scale, that together with $\xi_{\mathrm{PS}}$ is central to the physics of supercooled liquids and rules the relaxation within the RFOT picture.

The main physical ingredients of RFOT are the surface tension $\Upsilon$ and the configurational entropy density $s_{c}(T)$. These two quantities are defined in the range of temperature between the Kauzmann point $T_{K}$ and the mode-coupling crossover $T_{\mathrm{MCT}}$, where multiple amorphous phases are present. The former is a measure of the extra free-energy cost paid when two different amorphous phases are in contact through a common surface. The latter quantifies the multiplicity of amorphous phases in which the liquid can freeze. The mosaic state results from the competition between the configurational entropy gain due to local fluctuations between all possible amorphous states (all different type of "tiles" of the mosaic) and the surfaceenergy loss due to the mismatch at the boundary between 
two amorphous states. A lot of analytical and numerical works have been devoted to characterize the spatial extent of the tiles of the mosaic, which we call here cooperative rearranging regions (CRRs) by extending the original definition of Adam and Gibbs [13] to the RFOT context. Very few investigations have instead focused on their interfaces, for which no clear picture has yet arisen. Analytical studies based on Kac models describe these interfaces as flat [14,15], whereas scaling arguments suggest that they wander over a size comparable to the one of CRRs [2]. Numerical works suggest an intermediate scenario where these interfaces wander similarly to domain walls in disordered magnets $[7,16,17]$. Their width has been directly measured and shown to grow mildly when temperature is lowered in recent numerical simulations on pinned particle systems $[8,11,18,19]$, and in first experiments obtained by using optical traps in colloidal liquids [20]. The physical reason for this growth is still to be elucidated and basic questions remain unanswered. How much do these interfaces fluctuate? How do their fluctuations depend on temperature; in particular, do interfaces become rougher or flatter approaching the glass transition? How does their width compare with the other characteristic length, the point-to-set length, which measures the spatial extent of CRRs? In order to answer all these questions and fully characterize the real-space structure of supercooled liquids and the mosaic state advocated by RFOT theory, it is crucial to develop a complete theory of fluctuating interfaces between amorphous states.

In this work, we develop such a theory, obtain detailed predictions, and provide explanations for previous numerical findings. We show that interfaces are rough because they are pinned by self-induced disorder and that they are characterized by wandering exponents identical to the ones of domain walls in the random field Ising model (RFIM), thus strengthening the relationship between supercooled liquids and the RFIM found in Refs. [21-25]. These results allow us to establish that supercooled liquids are characterized by two different static lengths, $\xi_{\mathrm{PS}}$ and $\xi_{\perp}$, which measure, respectively, the spatial extent and the fluctuations of the shape of CRRs. Their scaling with respect to $s_{c}$ is different: $\xi_{\mathrm{PS}} \propto s_{c}(T)^{-1 /(3-\theta)}$ with $\theta>1$, whereas $\xi_{\perp} \propto s_{c}(T)^{-1 / 2}$. Therefore, when $s_{c}$ decreases approaching the glass transition, at $T_{K}$, boundary fluctuations grow but less strongly than the linear size. The resulting shape of CRRs is shown pictorially in Fig. 1. A different scenario is expected close to $T_{\mathrm{MCT}}$, where $s_{c}$ is finite and the two length scales could become comparable. In this case, the roughening of interfaces could have a direct influence on $\xi_{\mathrm{PS}}$ and on its scaling exponent $\theta$.

In order to study amorphous interfaces in three-dimensional systems, we adapt the theoretical protocol that has been used to characterize the size of CRRs [4]. We focus on equilibrated configurations $\mathcal{C}^{\prime}$ constrained to have a high overlap behind an infinite plane with a reference equilibrium configuration $\mathcal{C}$. This can be operatively realized by

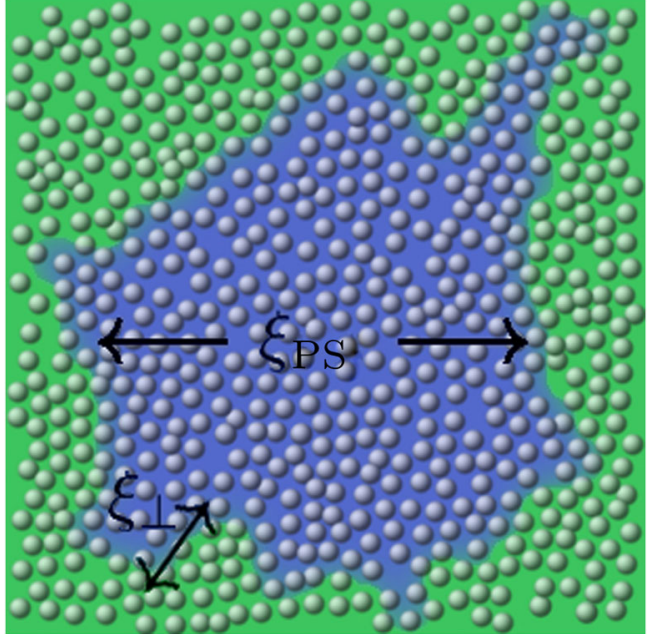

FIG. 1. Illustration of a two-dimensional cut of a cooperative rearranging region: the linear spatial extent is of the order $\xi_{\mathrm{PS}}$, whereas the external shape is rough and fluctuating over the length scale $\xi_{\perp}$.

taking an equilibrium configuration, pinning all particles behind a plane, and resampling the configuration of the remaining free particles; see Fig. 2 for a pictorial representation of this protocol. The precise way to define the overlap does not matter. Several were proposed in the literature (see, e.g., Ref. [7]): they all share the same physical ingredient; i.e., they display a high value when configurations resemble each other on a resolution scale larger than the short-time motion and smaller than the interparticle distance. Because of the pinning procedure, the overlap field between $\mathcal{C}$ and $\mathcal{C}^{\prime}$ is high close to the plane and reaches a low value, characteristic of bulk behavior, far from it. We define the amorphous interface for a given $\mathcal{C}^{\prime}$ as the surface separating the high overlap region from the low

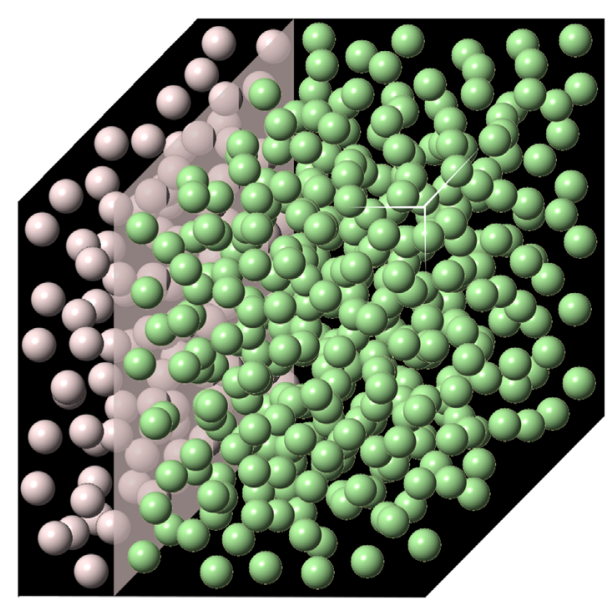

FIG. 2. Pictorial representation of the protocol used to study amorphous interfaces: pink particles on the left of the plane are pinned and green ones on the right are reequilibrated in the presence of this amorphous boundary condition. 
overlap one. Within RFOT theory, the physics behind the formation of amorphous interfaces is similar to wetting [26]: on the one hand, it is favorable for the system to change amorphous state (or CRRs) beyond the plane because this allows a net gain in configurational entropy $s_{c}$. On the other hand, this leads to a free-energy loss due to the surface tension $\Upsilon$. However, since the loss term scales as the surface, whereas the gain term scales as the volume, the former cannot counterbalance the latter and the drop in the overlap field is always favorable. Understanding how this takes place and how the resulting amorphous interfaces fluctuate is one of the main aims of this work. Note that if we had considered a curved wall, or a cavity, the average position of the interface and its fluctuations (encoded in correlation functions) would have also been a function of $\xi_{\perp} / R$, where $R$ is the curvature radius. Since the limit $\xi_{\perp} / R \rightarrow 0$ has no reasons to be singular, our analysis holds also more generally, as long as $R / \xi_{\perp} \ll 1$. In particular, since as we show $\xi_{\mathrm{PS}} \gg \xi_{\perp}$ when $s_{c}$ is small, it holds for CRRs close to the glass transition. The physical reason is that the curvature of CRRs is negligible on the scale over which interfaces fluctuate. On this scale the approximation of considering that the average profile of interfaces is flat and infinite, i.e., taking the overlap high behind an infinite plane, is justified. In the following, we focus on only the temperature regime below the mode-coupling transition temperature $T_{\mathrm{MCT}}$, where high and low overlap states are well defined and it makes sense to use the concept of configurational entropy. In the conclusion, we come back to the regime close to $T_{\mathrm{MCT}}$.

As noticed in several works, see Refs. $[23,27,28]$ and references therein, the overlap plays the role of an order parameter and the configurational entropy acts like a field favoring the low overlap state. Consequently, the situation is similar to the case of the ferromagnetic Ising model in a field $H$ below the critical temperature. The counterparts of high and low overlaps are positive and negative magnetizations, whereas the counterpart of the configurational entropy is the (negative) magnetic field $H$ [29]. This analogy has been proven to be instructive in understanding the physics of glass-forming liquids [23,28]. Consequently, we start our analysis by discussing the physical picture one obtains from it. Within this framework, pinning particles behind a wall amounts to forcing all spins behind a wall to point up. In this way, one induces an interface between the positively magnetized region close to the wall and the negative magnetized region favored by $H$ far from the wall. It is well known that in this case the effective Hamiltonian for the interface position $h(\mathbf{x})$ ( $h$ is the distance between the interface and the plane at position $\mathbf{x}$ in the $d-1$ planar dimensional space) reads [30]

$$
\mathcal{H}[h(\mathbf{x})]=\int d \mathbf{x}\left[\sigma \frac{(\nabla h)^{2}}{2}+H h(\mathbf{x})\right],
$$

where $\sigma$ is the surface-energy cost. The statistics of the interface is obtained by integrating over all interface configurations weighted by their corresponding Boltzmann weight with the constraint $h(\mathbf{x}) \geq 0$. At zero temperature $\mathcal{H}$ is minimized by choosing $h(\mathbf{x})=0$ for all $\mathbf{x}$; i.e., the interface is flat and stuck on the plane. For finite temperatures the interface fluctuates to gain entropy. In two dimensions the interface is a line and the functional integral can be mapped into a quantum mechanical problem that can be solved exactly [26]. One finds that it is entropically favorable for the interface to wander over a length $\xi_{\perp} \propto H^{-1 / 3}$ perpendicular to the plane and a length $\xi_{\|} \propto H^{-2 / 3}$ parallel to the plane. In three dimensions the wandering is logarithmic only, and in four and higher dimensions the interface is flat [31]. In conclusion, the analogy with the ferromagnetic Ising model suggests that $3 \mathrm{D}$ amorphous interfaces are essentially almost flat and $\xi_{\perp}$ diverges logarithmically with $s_{c}$. A previous analysis based on Kac models also led to a similar conclusion: interfaces are flat and characterized by $\xi_{\perp} \propto-\ln s_{c}$ (for energetic reasons) $[14,15]$. However, a crucial physical ingredient has not been taken into account yet: self-induced quenched disorder. The specific reference configuration naturally introduces quenched randomness in the problem, which plays a very important role in the physics of supercooled liquids, as already shown in Refs. [21-25]. Note that in a supercooled liquid there is no quenched disorder: it is the configuration from which the system has to escape in order to flow that plays the role of $\mathcal{C}$, i.e., of self-induced disorder. As it is known for random manifolds in random environments, disorder leads to a huge enhancement of the wandering of the interface, so large that thermal fluctuations become completely irrelevant. It is reasonable to expect that a similar phenomenon could also take place for amorphous interfaces. Indeed, by using replica field theory we show that the Hamiltonian governing the long-wavelength fluctuations of amorphous interfaces is given by Eq. (1) plus a random potential term $\int d \mathbf{x} V_{R}(h(\mathbf{x}), \mathbf{x})$, whose statistical properties are the same ones found for interfaces in the random field Ising model. The scaling theory of the RFIM then allows us to work out the behavior of the wandering length $\xi_{\perp}$, which we find to diverge as the square root of $1 / s_{c}$ in three dimensions. In the following, we derive the mapping to the RFIM. The resulting effective action for amorphous interfaces and the corresponding scaling theory for $\xi_{\perp}$ are presented in Sec. III.

\section{DERIVATION AND MAPPING TO THE RFIM}

As anticipated in the Introduction, in order to study amorphous interfaces we focus on equilibrated configurations $\mathcal{C}^{\prime}$ constrained to have a high overlap behind an infinite plane with a reference equilibrium configuration $\mathcal{C}$. This is realized by taking an equilibrium configuration, pinning all particles behind a plane, and resampling the configuration of the remaining free particles; see Fig. 2. The starting point of our analysis is considering the 
statistical field theory for the overlap field $p(z, \mathbf{x})$, which is a function of the distance $z$ from the fixed plane and of the coordinates $\mathbf{x}$ along the plane. This field measures the similarity between two equilibrium configurations: the first is free, whereas the second is constrained to coincide with the first behind the chosen reference plane. This boundary condition leads naturally to the existence of an interface, whose position along the $z$ axis, $h(\mathbf{x})$, corresponds to the region in space where $p(z, \mathbf{x})$ jumps from the high value enforced by the constraint close to the plane to the low value favored by the configurational entropy. Our aim in the following is to obtain the effective field theory on $h(\mathbf{x})$ starting from the one on $p(z, \mathbf{x})$. Following previous works that derived an effective interface Hamiltonian [30], we assume that the most relevant configurations of $p(z, \mathbf{x})$ are the ones corresponding to a single interface positioned in $h(\mathbf{x})$. This is natural since having more than one interface is unlikely. Consequently, henceforth we focus only on configurations $p^{h}(z, \mathbf{x})=q_{E A}$ for $0<z<h(\mathbf{x})$ and zero for $z>h(\mathbf{x})$, where $q_{E A}$ is the typical overlap of two configurations in the same amorphous state (it is associated with the local Debye-Waller factor characterizing molecular motion in the glass-forming liquid). We neglect the smoothness of the decrease from one to $q_{E A}$ just after the wall and from $q_{E A}$ to zero at $z \simeq h(\mathbf{x})$. Both simplifications are inessential to establish the effective field theory of $h(\mathbf{x})$, as we discuss later [32]. The effective Hamiltonian $\mathcal{H}_{R}[h(\mathbf{x})]$ is obtained by evaluating the action for the overlap field for $p=p^{h}(z, \mathbf{x})$ :

$$
\mathcal{H}_{R}[h(\mathbf{x})]=\mathcal{S}\left[p^{h}(z, \mathbf{x}) \mid \mathcal{C}\right],
$$

where we make explicit the dependence on the reference equilibrium configuration $\mathcal{C}$ that introduces the quenched disorder. In order to show that $\mathcal{H}_{R}[h(\mathbf{x})]$ coincides with Eq. (1) plus a random potential term, $\int d \mathbf{x} V_{R}(h(\mathbf{x}), \mathbf{x})$, whose variance is the one characteristic of domain walls in the RFIM, we compute the average and the variance of $\mathcal{H}_{R}[h(\mathbf{x})]$. We show that the former is equal to $\mathcal{H}[h(\mathbf{x})]$ and the latter, $\overline{\left\{\left(\mathcal{H}_{R}\left[h_{1}(\mathbf{x})\right]-\mathcal{H}\left[h_{1}(\mathbf{x})\right]\right)-\left(\mathcal{H}_{R}\left[h_{2}(\mathbf{x})\right]-\mathcal{H}\left[h_{2}(\mathbf{x})\right]\right)\right\}^{2}}$, is proportional to the volume $\mathcal{V}_{h_{1}, h_{2}}$ of the space embedded by the two interfaces described by $h_{1}(\mathbf{x})$ and $h_{2}(\mathbf{x})$. This is indeed the result expected for the RFIM case, where the correlator of the random potential is $\overline{V_{R}\left(h_{1}(\mathbf{x}), \mathbf{x}\right) V_{R}\left(h_{2}(\mathbf{x}), \mathbf{x}\right)} \propto\left|h_{1}\left(\mathbf{x}_{1}\right)-h_{2}\left(\mathbf{x}_{2}\right)\right| \delta\left(\mathbf{x}_{1}-\mathbf{x}_{2}\right)$ [33].

The computation of the cumulants of $\mathcal{H}_{R}[h(\mathbf{x})]$ is performed by introducing $n$ different copies (or real replicas) of the system in the presence of the same "disorder" $\mathcal{C}$ and averaging the replicated system over $\mathcal{C}$. Following Ref. [25], we define the action of the replicated system by the identity

$$
\exp \left(-\mathcal{S}_{r}\left[\left\{p_{a}\right\}\right]\right)=\overline{\exp \left(-\sum_{a} \mathcal{S}\left[p_{a}(h, \mathbf{x}) \mid \mathcal{C}\right]\right)^{\mathcal{C}}}
$$

where $a \in[0, n]$. The action $\mathcal{S}_{r}\left[\left\{p_{a}\right\}\right]$ generates all the cumulants of $\mathcal{S}[p(z, \mathbf{x}) \mid \mathcal{C}]$ through the equation

$$
\begin{aligned}
\mathcal{S}_{r}\left[\left\{p_{a}\right\}\right]= & \sum_{a} S_{1}\left[p_{a}\right]-\frac{1}{2} \sum_{a, b} S_{2}\left[p_{a}, p_{b}\right] \\
& +\frac{1}{3 !} \sum_{a, b, c} S_{3}\left[p_{a}, p_{b}, p_{c}\right] \cdots,
\end{aligned}
$$

where

$$
S_{1}[p]=\overline{\mathcal{S}[p \mid \mathcal{C}]^{\mathcal{C}}}
$$

and

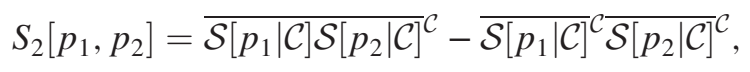

and so on.

In order to extract $S_{1}[p]$ from $\mathcal{S}_{r}\left[\left\{p_{a}\right\}\right]$, one considers all replicas equal, i.e., $p_{a}=p \forall a$, and picks from $\mathcal{S}_{r}\left[\left\{p_{a}\right\}\right]$ the term linear in $n$ since $S_{2}, S_{3}, \ldots$ are, respectively, of the order $n^{2}, n^{3}$, etc. Similarly, in order to obtain $S_{2}\left[p_{1}, p_{2}\right]$ one subdivides all replicas into two groups, such that $p_{a}$ is equal to $p_{1}$ and $p_{2}$ for replicas, respectively, belonging to the first and the second group. In this case, $S_{2}\left[p_{1}, p_{2}\right]$ is simply the part of the action proportional to $n_{1} n_{2}$ and can be, therefore, easily selected in the limit $n_{1}, n_{2} \rightarrow 0$. The technical procedure to follow in order to compute $\mathcal{S}_{r}\left[\left\{p_{a}\right\}\right]$ was derived in Ref. [25]. In the following we just quote the final result: $\mathcal{S}_{r}\left[\left\{p_{a}\right\}\right]$ is obtained as the free energy $\mathcal{L}$ of the replicated field theory for $n+1$ copies $\alpha \in[0, n]$ of the system, in which one fixes the overlaps $q_{\alpha 0}$ with the reference configuration labeled by $\beta=0$ to be equal to $p_{a}$ and integrates out all the others:

$$
\mathcal{S}_{r}\left[\left\{p_{a}\right\}\right]=-\log \int \prod_{\langle\alpha \beta\rangle} \mathcal{D} q_{\alpha \beta} \prod_{a=1}^{n} \delta\left[q_{a 0}-p_{a}\right] e^{-\mathcal{L}\left[\left\{q_{\alpha \beta}\right\}\right]} .
$$

As in Ref. [25], the integration is performed by saddle point (a more careful evaluation of the functional integral is not expected to give rise to any qualitative change). We use as action of the replicated field theory the Landau one proposed in Ref. [34] for supercooled liquids approaching the glass transition:

$$
\begin{aligned}
\mathcal{L}\left[\left\{q_{\alpha \beta}\right\}\right]= & \frac{E_{0}}{k_{B} T} \int_{z \mathbf{x}}\left\{\frac{c}{2} \sum_{\alpha \neq \beta}\left[\partial_{z \mathbf{x}} q_{\alpha \beta}(z, \mathbf{x})\right]^{2}+\sum_{\alpha \neq \beta} v\left(q_{\alpha \beta}\right)\right. \\
& \left.-\frac{u}{3} \sum_{\alpha \neq \beta \neq \gamma} q_{\alpha \beta}(z, \mathbf{x}) q_{\beta \gamma}(z, \mathbf{x}) q_{\gamma \alpha}(z, \mathbf{x})\right\},
\end{aligned}
$$

where it has been defined $v(q)=t q^{2} / 2-(u+w) q^{3} / 3+$ $y q^{4} / 4$, the main temperature dependence is in 
$t \sim k_{B}\left(T-T_{0}\right) / E_{0}, E_{0}$ is the liquid's energy scale, and $T_{0}$ is a constant. This choice of Landau functional encodes the ingredients that have been understood to be the key ones in recent years. The choice of $v(q)$ is such that $v(q)+u q^{3} / 3$ develops a secondary minimum below $T_{\mathrm{MCT}}$ in correspondence to the value $q=q_{E A}$. The height of this minimum is the configurational entropy $s_{c}(T)$. These features have been found first in mean-field models [27] and recently confirmed in numerical simulation of supercooled liquids [35-38]. The replica structure of the third term, that couples replicas, arises naturally in all mean-field models (see, e.g., Ref. [27]) and, hence, it has to be included. Our analysis holds for general functionals sharing these properties; in the following, we focus on Eq. (3) for concreteness.

In order to compute the first cumulant we take $p_{a}=p^{h}(z, \mathbf{x})$. Because of the resulting replica symmetry we solve the saddle point equations assuming $q_{a b}=q$ $\forall a \neq b$ and $n \rightarrow 0$. We first consider the case of a flat interface, i.e., $h(x)=h, p^{h}(z, \mathbf{x})=p(z)=q_{E A} \theta(h-z)$, where the latter is the Heaviside function. In this case $q$ depends only on $z$ and its saddle point equation reads

$$
c \partial_{z}^{2} q(z)-v^{\prime}(q(z))-2 u q(z)^{2}+u q_{E A}^{2} \theta(h-z)=0 .
$$

By numerically solving this Eq. [39], we find that an interface profile for $p^{h}$ induces a similar interface profile $q^{h}(z)=I(z-h)$, where $I$ is constant until $z=h$ and then decreases rapidly to zero; see Fig. 3. This physically makes sense since replicas that are forced to have a high overlap with a reference configuration within a distance $h$ from the wall are expected to also have a high cross-overlap. By plugging these profiles into the replicated action and focusing on the term proportional to $n$, one naturally finds two contributions, one that is proportional to the volume between the wall and the interface and another one, independent of the interface position, that scales as the surface [40]:

$$
\begin{aligned}
\overline{\mathcal{H}_{R}[h]} & \simeq \int d \mathbf{x}\left\{h\left[v\left(q_{E A}\right)+u q_{E A}^{3} / 3\right]+\Sigma\right\} \\
& =\int d \mathbf{x} s_{c} h+\Sigma L^{d-1},
\end{aligned}
$$

where $\Sigma$ is the cost per unit surface of creating an interface and $L^{d-1}$ is the wall surface. In order to compute $\Sigma$ correctly one should take into account an optimized and smooth form of the interface along the $z$ direction and possibly replica symmetry breaking [34]. We do not need to worry about this complication since the term linear in $h$, the one we are interested in, is independent of it: $\Sigma L^{d-1}$ is just an additional constant and does not play any role for the interface fluctuations. Let us now also include in the analysis longwavelength fluctuations of $h(x)$. Simple arguments show that in this case $q(z, \mathbf{x})$ has to follow "adiabatically" the profile of $p^{h}(z, \mathbf{x})=q_{E A} \theta[h(\mathbf{x})-z]$; i.e., $q(z, \mathbf{x})=I[z-$ $h(\mathbf{x})]$ up to subleading corrections in gradients of $h(\mathbf{x})$. By plugging this expression in the action, one finally obtains that $\overline{\mathcal{H}_{R}[h]}$ at large length scales and for $h \gg 1$ is precisely equal to $\mathcal{H}[h]$ defined in Eq. (1), with $\sigma=c$ and $H=s_{c}$, plus a constant term equal to $\Sigma$ times the wall surface.

Having obtained the first part of our technical results, we now turn to the study of the fluctuations of $\mathcal{H}_{R}[h]$. As discussed above, we have to consider two groups of replicas having an overlap profile with the reference configuration $p^{h_{1}}$ and $p^{h_{2}}$, respectively. As before, we start by focusing on flat interfaces positioned at $h_{1}$ and $h_{2}$. Without loss of generality we consider $h_{1}<h_{2}$. By writing the saddle point equation on $q_{a b}$ for $n_{1}, n_{2} \rightarrow 0$, one finds that the overlaps between replicas of the same group, $q^{h_{1}}$ and $q^{h_{2}}$, satisfy Eq. (4), where the role of $p^{h}$ is played by $p^{h_{1}}$ and $p^{h_{2}}$, respectively. The overlap $q_{12}$ between replicas of different groups satisfies the equation

$$
\begin{aligned}
c \Delta q_{12}= & v^{\prime}\left(q_{12}\right)-u q_{E A}^{2} \theta\left(h_{1}-z\right) \theta\left(h_{2}-z\right) \\
& +u q_{12}\left[q\left(z-h_{1}\right)+q\left(z-h_{2}\right)\right] .
\end{aligned}
$$

Our numerical solutions [41] show that $q_{12}$ assumes a profile very similar to $p^{h_{1}}$, i.e., the interface profile closer to the wall, and to $q^{h_{1}}: q_{12}$ is equal to $q_{E A}$ for $z<h_{1}$ and has a sharp drop to zero just after. This result, evident from Fig. 3 , is the key ingredient in the following calculation. Indeed, by plugging all the overlap profiles in the replicated action one finds that the second cumulant $S_{2}\left[p_{1}, p_{2}\right]$ reads

$S_{2}=-2 \int_{z \mathbf{x}}\left\{2 v\left(q_{12}\right)-2 u p^{h_{1}} p^{h_{2}} q_{12}+u q_{12}^{2}\left(q^{h_{1}}+q^{h_{2}}\right)\right\}$.

Using this result and in the limit $h_{1} \gg 1, h_{2} \gg 1$, $h_{1}-h_{2} \gg 1$, we obtain that the variance of the fluctuations $\delta \mathcal{S}[p \mid \mathcal{C}]=\mathcal{S}[p \mid \mathcal{C}]-\overline{\mathcal{S}[p \mid \mathcal{C}]^{\mathcal{C}}}$ reads

$$
\begin{aligned}
& \overline{\left(\delta \mathcal{S}\left[p^{h_{1}} \mid \mathcal{C}\right]-\delta \mathcal{S}\left[p^{h_{2}} \mid \mathcal{C}\right]\right)^{2}} \mathcal{C} \\
& \quad=S_{2}\left[p^{h_{1}}, p^{h_{1}}\right]+S_{2}\left[p^{h_{2}}, p^{h_{2}}\right]-2 S_{2}\left[p^{h_{1}}, p^{h_{2}}\right] \\
& \quad=4 \mathcal{V}_{h_{1}, h_{2}}\left(\frac{u}{3} q_{E A}^{3}-s_{c}\right),
\end{aligned}
$$

where $\mathcal{V}_{h_{1}, h_{2}}$ is the volume embedded by the surfaces $h_{1}$ and $h_{2}$. As discussed previously, if one considers longwavelength fluctuations of $h_{1}(\mathbf{x}), h_{2}(\mathbf{x})$, the overlaps $q^{h_{1}}$, $q^{h_{2}}, q_{12}$ follow adiabatically the solutions obtained for flat profiles. By plugging the corresponding solutions into the action one finds that $S_{2}$ acquires an extra contribution due to the gradient terms, which can be neglected at leading order since it scales as the area of the interfaces and not as the volume $\mathcal{V}_{h_{1}, h_{2}}$. Consequently, result Eq. (8) also holds for nonflat interfaces. 
We conclude this section with a remark on the previous analysis. In our derivation we consider that the interface is well defined by focusing on the kink solution and we also neglect the possibility of overhangs (or other strong fluctuations). This can be justified, at least to some extent, recalling that the temperatures we are considering are effectively low with respect to the important temperature scales in the problem. In fact, simulations [35-38] have shown that around (or slightly above) $T_{\mathrm{MCT}}$ one finds phase coexistence of high and low overlap phase and Maxwell construction for the overlap free energy. Thus, in the regime we are interested in, below $T_{\mathrm{MCT}}$, interfaces are expected to be well-defined objects. Moreover, in the supercooled regime the temperature, multiplied by $k_{B}$, is smaller than the typical microscopic energy scale, which corresponds to the high temperature limit of the effective activation energy [42]. This means that supercooled liquids are effectively at low temperature and suggests that strong interface fluctuations can be neglected, as we do.

\section{EFFECTIVE HAMILTONIAN AND SCALING THEORY}

We now collect all previous results and write down the effective Hamiltonian governing the long-wavelength fluctuations of amorphous interfaces. It is a random functional of $h(\mathbf{x})$ whose average and variance are the ones computed previously. We have not computed higher cumulants, but these are not expected to be relevant: they correspond to the non-Gaussian character of the quenched disorder [43]. Hence, for simplicity, we take them equal to zero in the following. The final model for amorphous interfaces reads

$$
\begin{aligned}
\mathcal{H}_{R}[h(\mathbf{x})]= & \int d \mathbf{x}\left[\frac{c \nabla h^{2}}{2}+s_{c} h(\mathbf{x})\right]+\int d \mathbf{x} V_{R}(h(\mathbf{x}), \mathbf{x}) \\
& +\Sigma L^{d-1}
\end{aligned}
$$

where $V_{R}(h(\mathbf{x}))$ is a random Gaussian potential with zero mean and $\Sigma$ is independent of the shape of the interface profile. The variance of $V_{R}(h(\mathbf{x}))$ reads

$$
\begin{aligned}
& \overline{V_{R}\left(h_{1}\left(\mathbf{x}_{1}\right), \mathbf{x}_{1}\right) V_{R}\left(h_{2}\left(\mathbf{x}_{2}\right), \mathbf{x}_{2}\right)} \\
& \quad=\left(\frac{u}{3} q_{E A}^{3}-s_{c}\right)\left|h_{1}\left(\mathbf{x}_{1}\right)-h_{2}\left(\mathbf{x}_{2}\right)\right| \delta\left(\mathbf{x}_{1}-\mathbf{x}_{2}\right) .
\end{aligned}
$$

Note that the variance is positive, as it should be, since in the regime we are interested in, i.e., $T$ close to $T_{K}$, the configurational entropy $s_{c}$ is small. We consider for simplicity a delta correlated disorder, since introducing explicitly finite-range correlations would not alter our conclusions. In reality, the disorder is short but finite-range correlated over a length that coincides with the width of the interface [the microscopic range over which $q^{h}(z)$ decays from $q_{E A}$ to 0], as one can obtain repeating the previous replica analysis for $h_{1}-h_{2}$ not too large.
The model we end up with is identical to the one describing domain walls in the RFIM in the presence of an external field. We can therefore use previous insights developed in this case, in particular the scaling theory developed by Fisher $[31,44]$, to work out the behavior of amorphous interfaces. Following Refs. [31,44], we introduce the potential $W(\ell)$, which is the change in the free energy per unit area of the interface when the latter is constrained to have an average distance $\ell$ from the plane. The fluctuations of the interface are determined by the balance between two competing mechanisms which lead to two different contributions to $W(\ell)$. An interface closer to the wall leads to a gain of configurational entropy and hence to an effective attractive interaction $W_{a}(\ell)$. In fact, the transition from the high to the low overlap state at a distance $\ell$ from the wall leads to a free-energy density gain (per unit surface) equal to $W_{a}(\ell)=W_{a}(0)+s_{c} \ell$. On the other hand, because of the random field disorder, the interface wanders over increasingly large length scales in order to find an optimized configuration that goes through favorable energetic regions, as it is known for the RFIM. Forcing the interface to wander on average no more than a distance $\ell$ from the wall induces a constraint and hence to a less optimized configuration, i.e., to a higher energy. As discussed in Ref. [31], this produces an effective repulsive potential between wall and interface (per unit surface) equal to $W_{r}(\ell)=W_{r}(0)+\frac{b}{\ell^{\tau}}$, where $b$ is a constant. The balance between these two mechanisms, i.e., the minimization of $W(\ell)$, sets the value of the typical distance of the amorphous interface from the wall: $\xi_{\perp} \propto s_{c}^{-1 /(\tau+1)}$. On length scales smaller than $\xi_{\perp}$, the effective attraction due to the configurational entropy can be neglected and the interface fluctuations are similar to the ones of a free interface [31]. Thus, moving along the plane one encounters over length scales $\xi_{\|} \propto \xi_{\perp}^{1 / \zeta}$ independent transverse fluctuations of the interface of the order $\xi_{\perp}$ ( $\zeta$ is the roughness exponent of free RFIM interfaces).

For the RFIM the Imry-Ma argument, validated by functional renormalization group analysis and numerical simulations, gives $\zeta=(5-d) / 3$ and $\tau=2 / \zeta-2=(2 d-4) /$ $(5-d)$ [31,33]. This leads to a width of amorphous interfaces scaling as

$$
\xi_{\perp} \propto s_{c}^{-(5-d) /(d+1)} .
$$

Consequently, we finally find that amorphous interfaces wander in three dimensions over a length $\xi_{\perp} \propto s_{c}^{-1 / 2}$.

Previous results underestimated fluctuations and accordingly found a more modest increase for $\xi_{\perp}$ : the analysis of Kac models in which both thermal and disorder fluctuations of the interface were neglected obtained $\xi_{\perp} \propto-\ln s_{c}$ $[14,15]$, whereas the real-space renormalization group study of Ref. [45] that took into account only thermal fluctuations predicted $\xi_{\perp} \propto\left(1 / s_{c}\right)^{1 / d}$. Our analysis, which 
treats all types of fluctuations, makes clear that the key physical mechanism to consider is self-induced disorder, which leads to much rougher and fluctuating amorphous interfaces and hence to a larger $\xi_{\perp}$, as shown in Eq. (11).

\section{PHYSICAL CONSEQUENCES, PREDICTIONS, AND COMPARISON TO NUMERICAL EXPERIMENTS}

A lot of theoretical and numerical studies have been devoted to unveiling static fluctuations and correlations in supercooled liquids [46]. The reason for this goes back to Adam and Gibbs, who introduced the idea that relaxation takes place through cooperative motion of statically correlated regions (CRRs). Within the Adam-Gibbs picture the relaxation time is associated to an energy barrier that grows with the size of the CRRs [13]. Refined approaches, developed more recently also trace back the increase of the relaxation time to statically correlated regions $[2,47]$. Probing CRRs means identifying and analyzing static fluctuations that incarnate the theoretical picture that arose from these analyses. This is difficult since standard twopoint correlation functions are ineffective. One has to focus instead on point-to-set (PS) correlations [4]. There are several possible general definitions of the PS correlation function $C_{\mathrm{PS}}$ [14], for example,

$$
C_{\mathrm{PS}}=\sum_{\mathcal{T}_{1}^{P} \mathcal{T}_{2}^{P}} q\left(\mathcal{T}_{1}^{P}, \mathcal{T}_{2}^{P}\right)\left\langle P\left(\mathcal{T}_{1}^{P} \mid \mathcal{C}^{S}\right) P\left(\mathcal{T}_{2}^{P} \mid \mathcal{C}^{S}\right)\right\rangle_{\mathcal{C}}
$$

In this formula $\mathcal{T}_{1}^{P}, \mathcal{T}_{2}^{P}$ denote the possible configurations at the point (or localized region) $P, \mathcal{C}^{S}$ the possible configurations at the set $S$, and $q\left(\mathcal{T}_{1}^{P}, \mathcal{T}_{2}^{P}\right)$ is the overlap measuring the similarity of $\mathcal{T}_{1}^{P}, \mathcal{T}_{2}^{P}$ (the average is over equilibrium configuration). The function $P\left(\mathcal{T}_{1}^{P} \mid \mathcal{C}^{S}\right)$, which

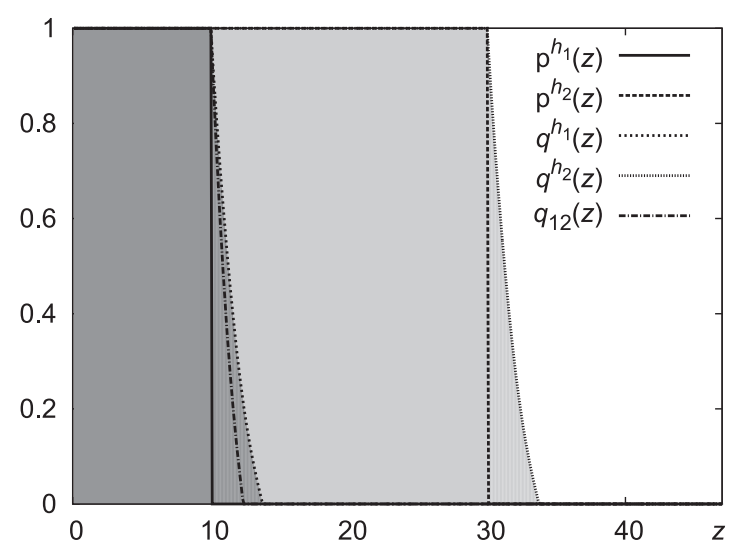

FIG. 3. Overlap profiles $p^{h_{1}}(z), p^{h_{2}}(z), q^{h_{1}}(z), q^{h_{2}}(z), q_{12}(z)$ for $h_{1}=10, h_{2}=30$ and $s_{c}=0$ (the unit of length is $\sqrt{c}$ ); as explained in the text, $q^{h_{1}}(z)=I\left(z-h_{1}\right)$. Note that $p^{h_{1}}(z)$ also represents the profile $p^{h}(z)$ obtained for a single replica. For the Landau action considered in the text, $q_{E A}=1$ when $s_{c}=0$. Similar results are obtained for a generic small value of $s_{c}$. is the conditional probability that the system has the configuration $\mathcal{T}_{1}^{P}$ in $P$ given that it has $\mathcal{C}^{S}$ in $S$, contains the essential information on static correlations. Whereas in standard second-order phase transitions it is enough to consider for $S$ just a localized region at distance $r$ from $P$, in supercooled liquids it is crucial to focus on an extended set.

Measuring the above correlation function could seem very challenging at first. The pinning protocol provides an operative way to do that. Indeed, one can rewrite the previous equation as

$$
C_{\mathrm{PS}}=\sum_{\mathcal{T}^{P}}\left\langle q\left(\mathcal{T}^{P}, \mathcal{C}^{P}\right) P\left(\mathcal{T}^{P} \mid \mathcal{C}^{S}\right)\right\rangle_{\mathcal{C}}
$$

This means taking an equilibrium configuration $\mathcal{C}$, pinning all particles in the set $S$, resampling the configuration $\mathcal{T}$ of the remaining free particles, and measuring the similarity at point $P$ between the configuration $\mathcal{T}$ and the reference one $\mathcal{C}$. Thus, pinning is the way to probe subtle static correlations of bulk liquids [48]. By considering different kinds of sets, one can probe different properties of CRRs. By considering as set $S$ all particles outside a cavity, one can characterize the spatial extent of amorphous order $\xi_{\text {PS }}$ and, hence, probe the linear size of the CRRs [4]; whereas by choosing as set $S$ all particles behind a plane, which is the procedure we focus on in this work, one gains information on the fluctuations of amorphous interfaces and, hence, accesses the length $\xi_{\perp}$ over which the shape of CRRs fluctuates [49].

The length $\xi_{\mathrm{PS}}$ is called point to set in the literature (even though it is just a special case of PS). Within RFOT it scales as $\xi_{\mathrm{PS}} \propto s_{c}^{-1 /(d-\theta)}$ [2]. The growth of $\xi_{\mathrm{PS}}$ is due to a different mechanism compared to the one associated to $\xi_{\perp}$ and, accordingly, the growth law is different. In three dimensions, within a Kac-like (instanton) approach one finds $\theta=d-1=2[34,50]$, as also suggested by some numerical simulations [16,17]. Although a conclusive result on the value of $\theta$ is still missing, all indications point toward a value of $\theta$ such that $1 /(d-\theta)$ is larger than $1 / 2$ in three dimensions (note, however, that the wetting argument of Kirkpatrick, Thirumalai, and Wolynes suggests $\theta=\frac{d}{2}=\frac{3}{2}$ [2] and at the same time $\xi_{\mathrm{PS}} \sim \xi_{\perp}$ ). Consequently, we do find as anticipated that CRRs, i.e., the regions over which the system is coherently in one amorphous state, are separated by interfaces that are rough but fluctuate less than the typical size of the regions; see Fig. 1 for a pictorial representation. This remains true for higher dimensions. Interestingly, for $d=2$ the exponents of $\xi_{\mathrm{PS}}$ and $\xi_{\perp}$ become equal (for $d=2$ we have $d-1=d / 2=(5-d) /$ $(d+1)=1)$. Note that $d=2$ is though to be the lower critical dimension for the glass transition since the selfinduced disorder prevents a transition from a low to high overlap phase in two dimensions; see Refs. [23,24].

The existence of two different static length scales governing the physics of supercooled liquids, $\xi_{\mathrm{PS}}$ and 
$\xi_{\perp}$, is a major fact to take into account in understanding the outcomes of simulations and experiments in glass-forming liquids, in particular when probing static correlations. Several lengths were proposed and measured in the literature and the relationship between them was blurred. Our findings makes clear that there exist two distinct length scales: one is the point to set measured using the spherical geometry, called $\xi_{\mathrm{PS}}$ in this work, and the other is the point to set measured using the wall geometry, originally introduced as penetration length, and that we call wandering length and denote $\xi_{\perp}$ in this work. We introduce this name in order to make explicit its physical origin and underline the relationship with the physics of random manifold in random media.

A first important consequence of our results is that they provide a natural explanation of the difference found in numerical simulations between the cavity and the wall geometry. In fact, it was shown that the lengths probed using these two settings grow in a different way, more mildly in the former case as indeed expected from our results $[19,51]$. Moreover, the decay of the overlap at the center of the cavity was found to be quite different from the decay of the overlap from the wall. In the first case, it becomes sharper at lower temperature (it can be fitted by an increasingly more compressed exponential) [7,12,19], whereas in the latter the form remains unchanged and exponential-like $[11,19]$. These differences have a natural explanation within the physical picture arising from our theory in which two growing static lengths, $\xi_{\perp}$ and $\xi_{\mathrm{PS}}$, intervene. Since the ratio $\xi_{\perp} / \xi_{\text {PS }}$ decreases by lowering the temperature, the CRRs are better and better defined on the scale $\xi_{\mathrm{PS}}$ and, therefore, the decay at the center of the cavity becomes indeed sharper and sharper at lower temperature. Instead, the decay of the overlap from the wall is governed by the length scale $\xi_{\perp}$ only (the point-to-set length does not play any role). In this case it is natural to expect scaling with respect to $\xi_{\perp}$, as also shown for manifolds in random media, and hence a decay that does not change form, in particular, does not become sharper by lowering the temperature. Note that another case in which taking into account the existence of two different static length scales is crucial to explain numerical data has been discussed recently in Ref. [52]: in order to rationalize the finite-size scaling of the specific heat for pinned systems in a cavity geometry, one needs to consider both $\xi_{\perp}$ and $\xi_{\mathrm{PS}}$. To test our scaling predictions it would be worth pushing further numerical simulations to obtain the dependence $\xi_{\perp}$ on $s_{c}$ for a realistic model of supercooled liquid. The RFIM character of amorphous interfaces that follows from our theory is already well supported by the numerical results of Refs. [16,17], which found a roughening exponent $\zeta \simeq 0.62 \pm 0.75$ and energy fluctuations scaling as $\ell^{2 \zeta}$. These two results compare extremely well with our predictions, which also lead to the same scaling of energy fluctuations and $\zeta=2 / 3 \simeq 0.66$.

\section{CONCLUSION}

In this work, we show that amorphous interfaces are rough in three dimensions and we obtain the scaling with the configurational entropy of the length scale over which they wander. Their statistical properties are identical to the ones of domain walls in random ferromagnets, a fact that strengthens even more the relationship between the physics of supercooled liquids and of the RFIM discussed in Refs. [21-25]. One of our major results is that there are two different static length scales governing the physics of supercooled liquids: the point-to-set length $\xi_{\mathrm{PS}}$, related to the spatial extent of CRRs, and the wandering length $\xi_{\perp}$, related to the fluctuations of their external shape. Our findings are in good agreement with previous numerical results, some of which were considered contradictory but find a natural explanation within our theory. We focus on the regime below $T_{\mathrm{MCT}}$, where CRRs are well formed and configurational entropy and interfaces are meaningful concepts. Approaching $T_{\mathrm{MCT}}$ we expect $\Upsilon$, and hence $c$, to decrease. This makes fluctuations more favorable. At a certain point, when they become so large that the long-wavelength theory with a simple square gradient term is not suitable anymore, the description of the interface we use might break down. We suspect that close to $T_{\text {MCT }}$ this leads to different scaling forms and is associated to the fractal, or stringy, nature of CRRs found in Ref. [53].

Finally, in view of the recent studies of the glass transition in high spatial dimensions [54-58], it is interesting to remark that our theory predicts a highly nontrivial dimensional dependence. In particular, we find $\xi_{\perp} \propto s_{c}^{-(5-d) /(1+d)}$ and, hence, an upper critical dimension $d_{u}=5$. In higher dimensions amorphous interfaces are flat, $\xi_{\perp}$ does not increase, and, hence, only one static growing length scale accompanies the glass transition. This is a striking change in the nature of the glass transition that would be worth testing numerically.

In conclusion, the predictions we obtain in this work provide a full characterization of the shape of cooperative rearranging regions in supercooled liquids. They are instrumental in interpreting, understanding, and devising new numerical simulations and experiments on static correlation in glass-forming liquids and clarify differences and relationships between the plethora of static lengths studied in recent years. There are several issues worth studying further with our formalism, which we leave for future work. One is whether in cases more accessible to experimental investigations, such as free surfaces instead of amorphous walls, the length $\xi_{\perp}$ can be probed [59]. Another is the effect of quantum fluctuations of the shape of CRRs. Previous works suggested that this is at the origin of the boson peak and the anomalies of low-temperature glasses [60].

\section{ACKNOWLEDGMENTS}

We thank T. Giamarchi, G. Parisi, G. Tarjus, and M. Tarzia for very useful discussions and W. Kob and 
P. G. Wolynes for useful comments on this manuscript. We acknowledge support from the ERC grants NPRGGLASS (G. B.), CRIPHERASY (C. C.) (No. 247328), and a grant from the Simons Foundation (\#454935, Giulio Biroli).

[1] G. Biroli and J.P. Garrahan, Perspective: The Glass Transition, J. Chem. Phys. 138, 12A301 (2013).

[2] T. R. Kirkpatrick, D. Thirumalai, and P. G. Wolynes, Scaling Concepts for the Dynamics of Viscous Liquids Near an Ideal Glassy State, Phys. Rev. A 40, 1045 (1989).

[3] M. Mézard and G. Parisi, A First-Principle Computation of the Thermodynamics of Glasses, J. Chem. Phys. 111, 1076 (1999).

[4] J.-P. Bouchaud and G. Biroli, On the Adam-GibbsKirkpatrick-Thirumalai-Wolynes Scenario for the Viscosity Increase in Glasses, J. Chem. Phys. 121, 7347 (2004).

[5] P. G. Wolynes and V. Lubchenko, Structural Glasses and Supercooled Liquids: Theory, Experiment, and Applications (John Wiley \& Sons, New York, 2012).

[6] A. Cavagna, T. S. Grigera, and P. Verrocchio, Mosaic Multistate Scenario versus One-State Description of Supercooled Liquids, Phys. Rev. Lett. 98, 187801 (2007).

[7] G. Biroli, J.-P. Bouchaud, A. Cavagna, T. S. Grigera, and P. Verrocchio, Thermodynamic Signature of Growing Amorphous Order in Glass-Forming Liquids, Nat. Phys. 4, 771 (2008).

[8] G. Parisi, On the Replica Scenario for the Glass Transition, arXiv:0911.2265.

[9] F. Sausset and G. Tarjus, Growing Static and Dynamic Length Scales in a Glass-Forming Liquid, Phys. Rev. Lett. 104, 065701 (2010).

[10] B. Charbonneau, P. Charbonneau, and G. Tarjus, Geometrical Frustration and Static Correlations in a Simple Glass Former, Phys. Rev. Lett. 108, 035701 (2012).

[11] L. Berthier and W. Kob, Static Point-to-Set Correlations in Glass-Forming Liquids, Phys. Rev. E 85, 011102 (2012).

[12] G. M. Hocky, T. E. Markland, and D. R. Reichman, Growing Point-to-Set Length Scale Correlates with Growing Relaxation Times in Model Supercooled Liquids, Phys. Rev. Lett. 108, 225506 (2012).

[13] G. Adam and J. H. Gibbs, On the Temperature Dependence of Cooperative Relaxation Properties in Glass-Forming Liquids, J. Chem. Phys. 43, 139 (1965).

[14] S. Franz, G. Semerjian et al., Dynamical Heterogeneities in Glasses, Colloids, and Granular Media (Oxford University Press, Oxford, England, 2011), p. 407.

[15] E. Zarinelli and S. Franz, Surface Tension in Kac Glass Models, J. Stat. Mech. (2010) P04008.

[16] C. Cammarota, A. Cavagna, G. Gradenigo, T. S. Grigera, and P. Verrocchio, Numerical Determination of the Exponents Controlling the Relationship between Time, Length, and Temperature in Glass-Forming Liquids, J. Chem. Phys. 131, 194901 (2009).

[17] C. Cammarota, A. Cavagna, G. Gradenigo, T. S. Grigera, and P. Verrocchio, Surface Tension Fluctuations and a New Spinodal Point in Glass-Forming Liquids, J. Stat. Mech. (2009) L12002.
[18] W. Kob, Ś. Roláan-Vargas, and L. Berthier, Spatial Correlations in Glass-Forming Liquids across the ModeCoupling Crossover, Phys. Procedia 34, 70 (2012).

[19] G. Gradenigo, R. Trozzo, A. Cavagna, T. S. Grigera, and P. Verrocchio, Static Correlations Functions and Domain Walls in Glass-Forming Liquids: The Case of a Sandwich Geometry, J. Chem. Phys. 138, 12 A509 (2013).

[20] S. Gokhale, K. Hima Nagamanasa, R. Ganapathy, and A. K. Sood, Growing Dynamical Facilitation on Approaching the Random Pinning Colloidal Glass Transition, Nat. Commun. 5, 4685 (2014).

[21] J. D. Stevenson, A. M. Walczak, R. W. Hall, and P. G. Wolynes, Constructing Explicit Magnetic Analogies for the Dynamics of Glass Forming Liquids, J. Chem. Phys. 129, 194505 (2008).

[22] S. Franz, G. Parisi, F. Ricci-Tersenghi, and T. Rizzo, Field Theory of Fluctuations in Glasses, Eur. Phys. J. E 34, 102 (2011).

[23] C. Cammarota and G. Biroli, Random Pinning Glass Transition: Hallmarks, Mean-Field Theory and Renormalization Group Analysis, J. Chem. Phys. 138, 12 A547 (2013).

[24] S. Franz and G. Parisi, Universality Classes of Critical Points in Constrained Glasses, J. Stat. Mech. (2013) P11012.

[25] G. Biroli, C. Cammarota, G. Tarjus, and M. Tarzia, RandomField-like Criticality in Glass-Forming Liquids, Phys. Rev. Lett. 112, 175701 (2014).

[26] M. E. Fisher, Walks, Walls, Wetting, and Melting, J. Stat. Phys. 34, 667 (1984).

[27] S. Franz and G. Parisi, Recipes for Metastable States in Spin Glasses, J. Phys. I (France) 5, 1401 (1995).

[28] F. Krzakala and L. Zdeborová, On Melting Dynamics and the Glass Transition. I. Glassy Aspects of Melting Dynamics, J. Chem. Phys. 134, 034512 (2011).

[29] More precisely, what plays the role of the magnetic field $H$ is the $\epsilon$-coupling introduced by Franz and Parisi [27] needed to induce a transition to the high overlap state, whereas the configurational entropy is the difference between the free energies corresponding to the high and low overlap states divided by $k_{B} T$. In the scaling regime we are interested in, close to $T_{K}$, these quantities are proportional: $\epsilon \propto k_{B} T_{K} s_{c} / q_{E A}\left(T_{K}\right)$, where $q_{E A}\left(T_{K}\right)$ is the nonergodic or Edwards-Anderson parameter measuring the fraction of frozen density fluctuations at $T_{K}$.

[30] H. W. Diehl, D. M. Kroll, and H. Wagner, The Interface in a Ginsburg-Landau-Wilson Model: Derivation of the Drumhead Model in the Low-Temperature Limit, Z. Phys. B 36, 329 (1980).

[31] M. E. Fisher, Statistical Mechanics of Membranes and Interfaces (World Scientific, Singapore, 2004).

[32] This smoothness should be taken into account in order to quantitatively reproduce the shape of the average interface profile.

[33] K. J. Wiese, The Functional Renormalization Group Treatment of Disordered Systems, Annales Henri Poincaré 4, 505 (2003).

[34] M. Dzero, J. Schmalian, and P. G. Wolynes, Replica Theory for Fluctuations of the Activation Barriers in Glassy Systems, Phys. Rev. B 80, 024204 (2009). 
[35] L. Berthier, Overlap Fluctuations in Glass-Forming Liquids, Phys. Rev. E 88, 022313 (2013).

[36] G. Parisi and B. Seoane, Liquid-Glass Transition in Equilibrium, Phys. Rev. E 89, 022309 (2014).

[37] L. Berthier and R. L. Jack, Evidence for a Disordered Critical Point in a Glass-Forming Liquid, Phys. Rev. Lett. 114, 205701 (2015).

[38] A. Ninarello, L. Berthier, and D. Coslovich, Structure and Dynamics of Coupled Viscous Liquids, Mol. Phys. 113, 2707 (2015).

[39] In the numerical integration the derivative of $q$ at the interface is chosen to optimize the action (actually maximize for reasons related to the $n \rightarrow 0$ limit).

[40] In Eq. (5) we only consider the leading term in $h$. The first correction to this behavior dependent on $h$ is of the form $\exp (-K h)$, where $K$ is a constant. This term, in the absence of quenched disorder, pushes the interface slightly away from the plane and leads to the result $\xi_{\perp} \propto-\ln s_{c}$ obtained in Refs. $[14,15]$. It can be neglected in the following since self-induced disorder leads to much stronger fluctuations and a more rapid increase of $\xi_{\perp}$ with $s_{c}$.

[41] In the numerical integration the derivative of $q_{12}$ at the interface is chosen to optimize the action (actually minimize for reasons related to the $n \rightarrow 0$ limit).

[42] M. L. Ferrer, C. Lawrence, B. G. Demirjian, D. Kivelson, C. Alba-Simionesco, and G. Tarjus, Supercooled Liquids and the Glass Transition: Temperature as the Control Variable, J. Chem. Phys. 109, 8010 (1998).

[43] L. Balents and D. S. Fisher, Large-n Expansion of (4- $\varepsilon$ )Dimensional Oriented Manifolds in Random Media, Phys. Rev. B 48, 5949 (1993).

[44] Edited by D. R. Nelson, T. Piran, and S. Weinberg, Statistical Mechanics of Membranes and Surfaces (World Scientific, Singapore, 1989), pp. 1-16.

[45] C. Cammarota, G. Biroli, M. Tarzia, and G. Tarjus, Renormalization Group Analysis of the Random FirstOrder Transition, Phys. Rev. Lett. 106, 115705 (2011).

[46] L. Berthier and G. Biroli, Theoretical Perspective on the Glass Transition and Amorphous Materials, Rev. Mod. Phys. 83, 587 (2011).

[47] G. Tarjus, S. A. Kivelson, Z. Nussinov, and P. Viot, The Frustration-Based Approach of Supercooled Liquids and the Glass Transition: A Review and Critical Assessment, J. Phys. Condens. Matter 17, R1143 (2005).

[48] Note that since all expressions of $C_{\mathrm{PS}}$ are just equilibrium averages, by trading ensemble averages with spatial averages, one obtains that the point-to-set correlations probed by $C_{\mathrm{PS}}$ are properties of a single equilibrium configuration of a very large system. This means that the pinning procedure is completely equivalent to one in which one selects all possible sets $S$ inside the system (e.g., all cavities) and then checks whether similar particle arrangements on $S$ are associated to similar particles arrangements on $P$. This equivalent (very cumbersome) procedure makes even clearer that point-to-set correlations are properties of bulk liquids.

[49] Note that we have considered for $S$ all particles behind an infinite plane for simplicity only. In order to make a more direct connection with the shape of CRRs we could have instead chosen for $S$ all particles in a cavity and then measured how much the overlap extends and fluctuates out of the cavity. When $\xi_{\perp} \ll \xi_{\mathrm{PS}}$, as it happens for $T$ close to $T_{K}$, the two choices lead to the same results.

[50] S. Franz, First Steps of a Nucleation Theory in Disordered Systems, J. Stat. Mech. (2005) P04001.

[51] W. Kob, S. Roldán-Vargas, and L. Berthier, Non-Monotonic Temperature Evolution of Dynamic Correlations in GlassForming Liquids, Nat. Phys. 8, 164 (2012).

[52] D. A. Martin, A. Cavagna, and T. S. Grigera, Specific Heat Anomaly in a Supercooled Liquid with Amorphous Boundary Conditions, Phys. Rev. Lett. 114, 225901 (2015).

[53] J. D. Stevenson, J. Schmalian, and P. G. Wolynes, The Shapes of Cooperatively Rearranging Regions in GlassForming Liquids, Nat. Phys. 2, 268 (2006).

[54] J. D. Eaves and D. R. Reichman, Spatial Dimension and the Dynamics of Supercooled Liquids, Proc. Natl. Acad. Sci. U.S.A. 106, 15171 (2009).

[55] P. Charbonneau, A. Ikeda, G. Parisi, and F. Zamponi, Glass Transition and Random Close Packing above Three Dimensions, Phys. Rev. Lett. 107, 185702 (2011).

[56] P. Charbonneau, A. Ikeda, G. Parisi, and F. Zamponi, Dimensional Study of the Caging Order Parameter at the Glass Transition, Proc. Natl. Acad. Sci. U.S.A. 109, 13939 (2012).

[57] B. Charbonneau, P. Charbonneau, Y. Jin, G. Parisi, and F. Zamponi, Dimensional Dependence of the Stokes-Einstein Relation and Its Violation, J. Chem. Phys. 139, 164502 (2013).

[58] P. Charbonneau, J. Kurchan, G. Parisi, P. Urbani, and F. Zamponi, Fractal Free Energy Landscapes in Structural Glasses, Nat. Commun. 5, 3725 (2014).

[59] S. Ashtekar, G. Scott, J. Lyding, and M. Gruebele, Direct Visualization of Two-State Dynamics on Metallic Glass Surfaces Well Below $T_{g}$, J. Phys. Chem. Lett. 1, 1941 (2010).

[60] V. Lubchenko and P. G. Wolynes, The Microscopic Quantum Theory of Low Temperature Amorphous Solids, Adv. Chem. Phys. 136, 95 (2007). 